\title{
Extreme geographical fixation of variation in the Plasmodium falciparum gamete surface protein gene $P f s 48 / 45$ compared with microsatellite loci
}

\author{
David J. Conway ${ }^{\mathrm{a}, *}$, Ricardo L.D. Machado ${ }^{\mathrm{b}}$, Balbir Singh ${ }^{\mathrm{c}}$, Patricia Dessert ${ }^{\mathrm{a}}$, \\ Zsuzsanna S. Mikes ${ }^{a}$, Marinete M. Povoa ${ }^{b}$, Ayoade M.J. Oduola ${ }^{d}$, Cally Roper ${ }^{a}$ \\ a Department of Infectious and Tropical Diseases, London School of Hygiene and Tropical Medicine, Keppel Street, London WC1E 7HT, UK \\ ${ }^{\mathrm{b}}$ Serviço de Parasitologia, Instituto Evandro Chagas, Belem, PA, Brazil \\ ${ }^{\mathrm{c}}$ Faculty of Medicine and Health Sciences, Universiti Malaysia Sarawak, Kota Samarahan 94300, Sarawak, Malaysia \\ ${ }^{\mathrm{d}}$ Postgraduate Institute of Medical Research and Training, College of Medicine, University of Ibadan, Ibadan, Nigeria
}

Received 27 October 2000; received in revised form 15 March 2001; accepted 20 March 2001

\begin{abstract}
Comparing patterns of genetic variation at multiple loci in the genome of a species can potentially identify loci which are under selection. The large number of polymorphic microsatellites in the malaria parasite Plasmodium falciparum are available markers to screen for selectively important loci. The $P f_{s} 48 / 45$ gene on Chromosome 13 encodes an antigenic protein located on the surface of parasite gametes, which is a candidate for a transmission blocking vaccine. Here, genotypic data from 255 P. falciparum isolates are presented, which show that alleles and haplotypes of five single nucleotide polymorphisms (SNPs) in the $P f s 48 / 45$ gene are exceptionally skewed in frequency among different $P$. falciparum populations, compared with alleles at 11 microsatellite loci sampled widely from the parasite genome. Fixation indices measuring inter-population variance in allele frequencies $\left(F_{\mathrm{ST}}\right)$ were in the order of four to seven times higher for $P f_{s} 48 / 45$ than for the microsatellites, whether considered (i) among populations within Africa, or (ii) among different continents. Differing mutational processes at microsatellite and SNP loci could generally affect the population structure at these different types of loci, to an unknown extent which deserves further investigation. The highly contrasting population structure may also suggest divergent selection on the amino acid sequence of Pfs $48 / 45$ in different populations, which plausibly indicates a role for the protein in determining gamete recognition and compatibility. (C) 2001 Elsevier Science B.V. All rights reserved.
\end{abstract}

Keywords: Plasmodium falciparum; Gametes; Pfs48/45; Genetics; Microsatellites; Selection

\section{Introduction}

A high density of informative microsatellite markers on all 14 chromosomes of the protozoan malaria parasite Plasmodium falciparum [1] should help studies to identify functionally important loci in the genome [2]. One approach is to identify loci with extreme statistical distributions of allele frequencies among populations. Exceptionally skewed frequency distributions may be

Abbreviations: SMM, stepwise mutation model; SNP, single nucleotide polymorphism.

* Corresponding author. Tel.: + 44-20-79272331; fax: + 44-2076368739 .

E-mail address: david.conway@1shtm.ac.uk (D.J. Conway). caused by directional selection for favoured alleles, whereas unusually even frequencies may be due to balancing selection maintaining different alleles [3,4]. Several asexual blood stage antigens of $P$. falciparum are apparently under selection which maintains allelic variation [5-9], and a detailed study of polymorphisms in the merozoite surface protein 1 gene (mspl) has prospectively identified a domain encoding a target of allele-specific protective immune responses [10].

In contrast to many antigens of the $P$. falciparum asexual blood stages, there is a relatively low level of sequence polymorphism in antigens of sexual gametocyte and gamete stages of the parasite. A major surface antigen of these stages is the protein Pfs48/45 [11], which has an unusual predicted disulphide bond-depen- 